\title{
Preface to Special Issue: Theory and Applications of Models of Computation (TAMC 2008-2009)
}

\author{
MANINDRA AGRAWAL ${ }^{\dagger}$, BARRY COOPER ${ }^{\ddagger}$ and ANGSHENG LI \\ ${ }^{\dagger}$ Department of Computer Science and Engineering, \\ Indian Institute of Technology Kanpur, India - 208016 \\ Email: manindra@iitk.ac.in \\ ${ }^{\ddagger}$ School of Mathematics, University of Leeds, \\ Leeds, LS2 9JT, United Kingdom \\ Email: pmt6sbc@leeds.ac.uk \\ ${ }^{\S}$ Institute of Software, Chinese Academy of Sciences, P. O. Box 8718, \\ Beijing, 100190, P. R. China \\ Email: angsheng@ios.ac.cn \\ Received 5 May 2010
}

The Theory and Applications of Models of Computation (TAMC) conference series is both international and interdisciplinary in character, bringing together researchers working in computer science, mathematics (especially logic) and the physical sciences. It is this, together with its predominantly computational and computability theoretic focus, that gives the series its special character.

TAMC 2008 and 2009 were, respectively, the 5th and 6th conferences in the series. The previous four meetings were held in Beijing (17-19 May 2004), Kunming, P. R. China (1720 May 2005), Beijing (15-20 May 2006) and Shanghai (22-25 May 2007). TAMC 2008 and 2009 were held in Xi'an (25-29 April 2008) and Changsha (18-22 May 2009), respectively. TAMC 2010, 2011 and 2012 will be held in Prague, Tokyo and Beijing, respectively. In particular, TAMC 2012 will be part of the 2012 Turing Year in China.

There were two plenary speakers at TAMC 2008, Bernard Chazelle and Cynthia Dwork, and two special sessions organised by Barry Cooper and Ying Jiang, on 'Models of Computation', and Jianer Chen, on 'Algorithms and Complexity', each containing four or five invited speakers, and 50 contributed papers accepted by the Programme Committee from the 192 papers submitted. At TAMC 2009, there were three plenary speakers, Leslie Valiant (Harvard University, U.S.A.), Moshe Vardi (Rice University, U.S.A.) and Matthew Hennessy (Trinity College, Ireland), and two special sessions organised by Barry Cooper, on 'Models of Computation', and Iyad A. Kanj, on 'Algorithms and Complexity', together with 39 papers selected by the Programme Committee from 86 submissions.

From amongst these contributions, we, as guest editors, have selected eleven of the best papers on the theme of computation and mathematical structures for inclusion in this special issue of Mathematical Structures in Computer Science.

The TAMC conference series arose naturally in response to important scientific developments affecting how we compute in the twenty-first century. At the same time, TAMC is already playing an important regional and international role, and promises to become a key contributor to the scientific resurgence seen throughout China and other 
parts of Asia. The excellence of the papers to be found here are fitting signifiers of this growing international involvement.

We would like to thank Giuseppe Longo and the members of the Editorial Board of Mathematical Structures in Computer Science and the Editors at Cambridge University Press for their encouragement and cooperation throughout the preparation of TAMC 2008 and 2009, and this special issue of the journal.

Of course, TAMC 2008 and 2009 would not have been possible without the support of our sponsors, and we therefore gratefully acknowledge their help in the realisation of this conference.

Manindra Agrawal

Barry Cooper

Angsheng Li

Guest Editors 